\title{
Akupunktur und transkutane Nerven- stimulierung bei der Nachbehandlung von Schlaganfällen
}

\author{
Johansson BB, Haker E, von Arbin M, Britton M, \\ Langstrom G, Terent A, Ursing D, Asplund K, for the \\ Swedish Collaboration on Sensory Stimulation After \\ Stroke: Acupuncture and transcutaneous nerve \\ stimulation in stroke rehabilitation. A randomized, \\ controlled trial. Stroke 2001;32:707-713
}

Background and Purpose: In small trials with control groups that receive no intervention, acupuncture has been reported to improve functional outcome after stroke. We studied effects of acupuncture and transcutaneous electrical nerve stimulation on functional outcome and quality of life after stroke versus a control group that received subliminal electrostimulation.

Methods: In a multicenter randomized controlled trial involving 7 university and district hospitals in Sweden, 150 patients with moderate or severe functional impairment were included. At days 5 to 10 after acute stroke, patients were randomized to 1 of 3 intervention groups: (a) acupuncture, including electroacupuncture; (b) sensory stimulation with high-intensity, low-frequency transcutaneous electrical nerve stimulation that induces muscle contractions; and (c) low-intensity (subliminal) high-frequency electrostimulation (control group). A total of 20 treatment sessions were performed over a 10-week period. Outcome variables included motor function, activities of daily living function, walking ability, social activities, and life satisfaction at 3-month and 1-year follow-up.

Results: At baseline, patients in each group were closely similar in all important prognostic variables. At 3-month and 1year follow-ups, no clinically important or statistically significant differences were observed between groups for any of the 
outcome variables. The 3 treatment modalities were all conducted without major adverse effects.

Conclusions: When compared with a control group that received subliminal electrostimulation, treatment during the subacute phase of stroke with acupuncture or transcutaneous electrical nerve stimulation with muscle contractions had no beneficial effects on functional outcome or life satisfaction.

\section{Kommentar - M. Lacour, Freiburg i.Br.}

\section{Klinische Einschätzung}

Es gibt sicherlich unterschiedliche Meinungen über die Nadelbehandlung von Patienten mit apoplektischem Insult. Bisher gibt es keinen allgemeinen Konsens oder akzeptierten Standard. Abweichend von der von Johansson et al. gewählten Akupunkturstrategie favorisieren wir eine Elektrostimulationsakupunktur über den Yin-Qiao-Mai und Yin-Wei-Mai bzw. über den Yang-Qiao-Mai und Yang-Wei-Mai, je nachdem ob die Spastik überwiegend an der Innen- oder Aussenseite der unteren oder oberen Extremität lokalisiert ist. Neben einer niederfrequenten Elektrostimulation kann auch eine mittelfrequente $50-\mathrm{Hz}$-Stimulation durchgeführt werden, die einen Ausgleich zwischen Qi und Blut fördern soll. Um einen Ausgleich zwischen der linken und rechten Körperhälfte zu schaffen, behandeln wir auf der nichtparetischen Seite die gekoppelten Öffnungspunkte der genannten aussergewöhnlichen Meridiane. Eine Elektrostimulationsakupunktur ist hier nicht erforderlich. Darüber hinaus kann die Akupunkturstrategie auch in einer kontrollierten Studie durch eine Behandlung gemäss der chinesischen Syndromdifferenzierung ergänzt werden. Ein apoplektischer Insult ist nach der chinesischen Medizin häufig auf eine Winderkrankung (Feng-Zheng) zurückzuführen. Eine durch äusseren Wind hervorgerufene Winderkrankung (Wai-Feng) spielt, wie auch durch andere Witterungseinflüsse hervorgerufene Syndrome (Hitze, Kälte, Nässe, Trockenheit) in der westlichen Welt eher eine untergeordnete Rolle. Eine wichtige Krankheitsursache ist hingegen der Innere Wind (Nei-Feng), der auch als Leberwind bezeichnet wird und z.B. durch heftig aufsteigendes Leber-Yang bei Leber-Yin-Leere oder Leber-Feuer bei Leber-Qi-Blockade entstehen kann. Diese innere Prädisposition kann allerdings durch psychische Bedingungen (insbesondere Wut und verdrängte Aggressionen), sowie durch eine, durch äussere Faktoren verursachte Erkrankungen (insbesondere ein ShaoYang-Syndrom) erst klinisch manifest werden. Weitere wichtige Krankheitsfaktoren sind innerer Schleim bei Milz-Qi-, oder Milz-Yang-Leere, sowie kombinierte Leere von Qi und Blut, die im Alter sehr häufig ist und nach der chinesischen Medizin durch komplexe Störungen der 5 Speicherorgane (und deren korrespondierenden Hohlorgane) verursacht wird. Die dargestellte Meridian-bezogene und konstitutionelle Therapie wird häufig durch chinesische Schädelakupunktur ergänzt, die in der vorliegenden Studie nicht angewendet wurde. Nach den Vorstellungen der chinesischen Medizin würde die Nadelbehandlung ausserdem durch chinesische Massagetechniken, Tai-Qi, Qi Gong und Phytotherapie ergänzt.

\section{Studienqualität}

Die folgenden Punkte sind bei der Studie positiv zu bewerten:

- Ethik: Zustimmung der Ethik-Kommission, Erhebung von unerwarteten Effekten.

- Studiendesign: Randomisierte (Blockrandomisierung), kontrollierte Studie.

- Ein- und Ausschlusskriterien, Selektionsprozess: Klar definierte Diagnosekriterien nach WHO und von Kriterien für den Grad der funktionelle Beeinträchtigung durch apoplektischen Insult. Klar definierte Ausschlusskriterien bezüglich neurologischer oder psychiatrischer Begleiterkrankungen, der Geschäftsfähigkeit und der gleichzeitigen Teilnahme an einer Behandlungsstudie.

- Zielkriterien: Klar definierte, validierte Testinstrumente. Hauptzielkriterium wurde genannt.

- Gruppenvergleich: Einheitliche konventionelle Therapie in allen Gruppen. Differenzierte Darstellung des Gruppenvergleichs unter Berücksichtigung bedeutsamer und prädisponierender Grunderkrankungen. Score-Werte für Haupt- und Nebenzielkriterien vor Therapiebeginn in allen Gruppen ausgeglichen. Erwartungshaltung in der Akupunkturgruppe gleich wie in der Kontrollgruppe die kein TENS erhielt.

- Intervention: Einheitliche Schulung der Therapeuten. Nur wenige und unbedeutende unerwartete Ereignisse/Nebenwirkungen.

- «Intention to treat»: Auswertung nach «intention to treat». Vollständigkeit des Follow-ups, «drop-outs»: Relativ wenige «drop-outs».

- Erhebung der Zielgrössen: Evaluation durch einen verblindeten Untersucher. «Allocation concealment» für Untersucher eingehalten.

Folgende Punkte müssen kritisch bewertet werden:

- Ethik: Unklarheit, ob eine schriftliche Einverständniserklärung eingeholt wurde.

- Studiendesign: Unklarheit, ob Patienten verblindet waren.

- Ein- und Ausschlusskriterien, Selektionsprozess: Selektionsprozess nicht detailliert dargestellt. Unklarheit, ob Patienten mit zerebralen Blutungen ausgeschlossen wurden. Unklarheit, ob Patienten mit schweren internistischen Begleiterkrankungen wie Herzinsuffizienz im NYHA-Stadium 3-4 oder anderen akuten Erkrankungen wie Myokardinfarkt ausgeschlossen wurden.

- Gruppenvergleich: Medikation im Gruppenvergleich nicht dargestellt. Keine Gleichverteilung bei koronarer Herzerkrankung, Vorhofflimmern und Normalbefunden im CT. In einem logistischen Regressionsmodell wurde bezüglich des Hauptzielkriteriums Vorhofflimmern als Determinante für ein schlechtes Outcome bestimmt. Differenzierung 
der CT-Befunde in Normalbefunde und ischämische Infarkte, nicht jedoch Aufschlüsselung der anderen Befunde (z.B. Blutung?). Erwartungshaltung in der TENS-Gruppe höher als in der Akupunkturgruppe.

- Intervention: Bezüglich der konventionellen Therapie, Unklarheit über die Höhe der Antikoagulation, insbesondere bei bestehendem Vorhofflimmern.

- Vollständigkeit des Follow-ups, «drop-outs»: Vollständige Durchführung des Follow-ups nicht nachprüfbar. Ergebnisse des Follow-ups für das Hauptzielkriterium nicht detailliert dargestellt.

- Erhebung der Zielgrössen: Unklarheit, ob die Untersucher bezüglich der Anwendung der Testinstrumente geschult und validiert wurden bzw. ob die Follow-up-Untersuchungen durch jeweils den gleichen Untersucher durchgeführt wurde.

\section{Präzision}

Gute Power (80\%).

\section{Zusammenfassung}

Die Studie besitzt eine ausreichende Power, um orientierende Aussagen über fehlende Therapieeffekte machen zu können. $\mathrm{Ob}$ die angewendete Akupunkturstrategie optimal gewählt wurde, lässt sich aus wissenschaftlicher Sicht derzeit nicht beurteilen. Nach empirischen Kriterien würden wir eine andere Therapieplanung favorisieren. Trotz vorhandener methodischer Mängel muss die Studienqualität insgesamt positiv bewertet werden. Demgegenüber sind wichtige Kriterien für den Gruppenvergleich aber nicht gleichmässig verteilt. So ist der Anteil der Patienten mit Vorhofflimmern in der Akupunkturgruppe am höchsten. Gleichzeitig wurde Vorhofflimmern als Determinante für ein schlechtes Outcome identifiziert. Die Ergebnisse der Studie sollten daher nur als Hinweis gewertet werden, dass die in der Studie angewendete Akupunkturstrategie gegenüber TENS oder einer Minimalbehandlung nicht überlegen ist. Aus der Studie können insbesondere keine pauschalen Aussagen abgeleitet werden, dass Akupunktur in der Behandlung von Patienten mit apoplektischem Insult nicht wirksam ist.

Kontaktadresse: Dr. Michael Lacour, Ambulanz für Naturheilverfahren und Umweltmedizin, Institut für Umweltmedizin und Krankenhaushygiene, Hugstetter Str. 55, D-79106 Freiburg, E-mail mlac@ iuk3.ukl.uni-freiburg.de.

Adresse für Sonderdrucke: Prof. Kjell Asplund, Dept. Medicine, University Hospital, SE-901 85 Umea, Sweden, E-mail kjell.asplund@medicin. umu.se. 